\title{
Investigación y educación ambiental: integración del conocimiento ecológico y del espacio geográfico en la costa del Río de la Plata, Uruguay
}

\author{
Elena Castiñeira Latorre ${ }^{1}$, Marila Lázaro ${ }^{2}$, Andrés Canavero ${ }^{1,3}$, Marcel Achkar ${ }^{4}$ \\ ${ }^{1}$ Sección Ecología Terrestre, Facultad de Ciencias (FC), Universidad de la República (UdelaR), \\ Uruguay,E-mail: elencasti@gmail.com \\ ${ }^{2}$ Unidad de Ciencia y Desarrollo, FC, UdelaR. \\ ${ }^{3}$ Center for Advanced Studies in Ecology \& Biodiversity y Departamento de Ecología, Pontificia \\ Universidad Católica de Chile. \\ ${ }^{4}$ Lab. Desarrollo Sustentable y Gestión Ambiental del Territorio, FC, UdelaR.
}

*Este artigo deve ser referido como segue:

Latorre E. C., Lázaro M., Canavero A., Achkar M. 2010. Investigación y educación ambiental: integración del conocimiento ecológico y del espacio geográfico en la costa del Río de la Plata Uruguay. Terræ Didatica, 6(2): $76-84<\mathrm{http}: / /$ www.ige.unicamp.br/ terraedidatica/>

\begin{abstract}
INVESTIGATION AND ENVIRONMENTAL EDUCATION: INTEGRATION OF THE ECOLOGICAL KNOWLEDGE AND THE GEOGRAPHIC SPACE AT THE COAST OF THE RÍO DE LA PLATA, URUGUAY. Facing the global recognition of an installed environmental crisis, Environmental Education (EA) arises as an engaged area for solving environmental problems. This is indispensable for a social transformation to revert problems towards a sustainable development. Environmental subjects require from science a bigger attention of extra-scientific elements implicit in the goals of knowledge and conservation of ecosystems, incorporating new dimensions that transcend, and may be cross-linked with, the scientific framework, and shared by society. In this work, we connect scientific results with educational proposals. These will be offered to the community through an educational portal online, being a new form of presenting and of broadcasting the scientific knowledge contributing to the environmental teaching.
\end{abstract}

KEYWORDS Investigation, Environmental Education, Knowledge Integration, Sustainable Development

RESUMEN Frente al reconocimiento global de una instalada "crisis ambiental", surge la Educación Ambiental (EA) como una de las áreas comprometidas en la resolución de problemas ambientales. Siendo indispensable para una transformación social con la cual revertir la problemática y transitar hacia un desarrollo sustentable. El tratamiento de las temáticas ambientales requiere por parte de la ciencia una mayor atención a elementos extra-científicos que se encuentran implicados en las metas del conocimiento y conservación de los ecosistemas, incorporando nuevas dimensiones que transciendan lo cientifico y que puedan ser interrelacionadas con, y compartidas por la sociedad. En particular, la ecología, como ciencia, permite abordar la problemática ambiental a través de sus diferentes escalas de análisis y consigue permear a través de la EA aportando en el manejo ambiental y la conservación. El presente trabajo plantea nuevas propuestas educativas basadas en los resultados obtenidos de una investigación científica. Éstas se ofrecerán a la comunidad a través de un portal educativo online generando una nueva forma de presentar, transponer $y$ difundir el conocimiento científico; conocimiento que pretende contribuir a la enseñanza ambiental.

PALABRAS-CLAVE Educación Ambiental, Desarrollo Sustentable, Integración de Conocimientos, Investigación. 


\section{Introducción}

El surgimiento de la "cuestión ambiental" como temática prioritaria y el reconocimiento de una "crisis ambiental" como un problema que afecta el destino de la humanidad ha sido reconocido fuertemente en las últimas décadas (Kates \& Parris 2003). El origen de los debates sobre las posibles causas y soluciones para revertir la instalada tendencia hacia la degradación ambiental se sitúa en las décadas del 60 y 70 (Martínez Alier 1995). Durante éste período se produce el nacimiento de distintas disciplinas y enfoques en relación al ambiente: la ética ambiental, la educación ambiental (EA), los ecologismos, que se nutrieron de las controversias sociales y la crítica creciente (de la academia pero también de los movimientos sociales) al poder excesivo y autoritario de la política, y de la ciencia y tecnología que, a pesar de su pretendida neutralidad, se mostraban asociadas al poder político y económico (González García et al. 1996, Bondolfi 2001).

Desde entonces diversas iniciativas se han generado para concientizar, educar y movilizar a los ciudadanos en pos del cuidado ambiental (González Gaudiano 2001, Artaraz 2002, Gutiérrez \& Pozo 2005, Gutiérrez \& Pozo 2006, Ferreira et al. 2007, Morais \& Silva 2007, Bustillo \& Martínez 2008). El surgimiento de la EA, como una de las áreas comprometidas en la resolución de problemas ambientales, se remonta a las diversas cumbres y convenciones internacionales que se llevan a cabo a partir de los años 70 (Feldmann \& Biderman Furriela 2001, Gough 2002, Casado \& Alonso Mielgo 2007).

La Conferencia de Naciones Unidas sobre el Medio Humano en Estocolmo, Suecia, en el año 1972, marca el inicio formal de la EA a nivel mundial. Posteriormente, en el año 1975, se formula en Belgrado el Programa Internacional de la UNESCO para la EA, en donde se define la categoría ambiente y los objetivos de la EA, además de señalar la complejidad del objeto de enseñanza y el necesario abordaje multidisciplinario (UNESCOPNUMA 1975). En el año 1977 en la Conferencia Intergubernamental convocada por la ONU en Tbilisi-Georgia, se discute la vinculación entre ambiente y los modelos de desarrollo, contextualizándose los problemas ambientales en el espacio y el tiempo (UNESCO 1977). Situando a la EA en un eje transversal se pretende comprender en un inicio el ambiente local, para posteriormente abordar lo nacional, regional y global, incluyendo la concepción de "responsabilidad intergeneracional". En 1987 se publica el informe "Nuestro Futuro Común”, elaborado por la Comisión Mundial sobre Ambiente y Desarrollo, el que define y propone el concepto de desarrollo sustentable (UN 1987). Desde ese momento el concepto, aceptado de forma generalizada por la ciencia, la política y las organizaciones sociales, se instala en las discusiones sobre el ambiente y el desarrollo económico. La Conferencia Mundial Río 92, reunión mundial de jefes de estado, planifica a escala internacional políticas ambientales y de desarrollo, mientras que se plantea una agenda internacional para los gobiernos conocida como la Agenda 21.

En la Agenda 21 se consagra un capítulo a la EA, considerándola indispensable para el cambio de actitudes y comportamientos hacia un desarrollo sustentable. Paralelamente a la reunión oficial de Río (1992) se realizaron convocatorias por parte de las ONGs y los movimientos sociales, como el Foro Global Ciudadano que, aunque menos publicitado, produjo varios documentos en los que se reforzaba la idea de que la EA debía ser un acto político basado en valores para la transformación social. En su declaración de principios (Tratado de EA para Sociedades Sustentables y Responsabilidad Global 1992) se lee:

La EA es un proceso de aprendizaje permanente, basado en el respeto a todas las formas de vida... tal educación afirma valores y acciones que contribuyen a la transformación humana y social y a la preservación ecológica. Ella estimula la formación de sociedades socialmente justas y ecológicamente equilibradas, que conserven entre sí una relación de interdependencia $y$ diversidad. Además, se define como una educación que "no es neutra sino ideológica".

Los citados hechos, que constituyen una parte del proceso fundacional de la EA y en el cual se articula el concepto de sustentabilidad, contienen una diversidad de paradigmas teóricos y de enfoques prácticos (Sauvé 1999, Bustillo \& Martínez 2008). Sin embargo, todas las tendencias comparten un sustrato común que reconoce la instalada crisis ambiental y la necesidad de soluciones (Costanza 2000, Achkar et al. 2005a, Bustillo \& Martínez 2008). El tratamiento de las temáticas ambientales requiere por tanto, por parte de la ciencia, una mayor atención 
a elementos extra-científicos que necesariamente se encuentran implicados en las metas del conocimiento y conservación de los ecosistemas (Gutiérrez \& Pozo 2005, Arocena et al. 2008).

Porlán (1999) se refiere a la necesidad de extraer de la investigación científica dimensiones que transciendan lo científico y que puedan ser interrelacionadas con, y compartidas por la sociedad. De esta forma la ciencia a través de sus diferentes disciplinas científicas puede proporcionarle a la sociedad información esencial para un razonamiento práctico (Barreiro et al. 1996). En particular, la ecología, como ciencia, permite abordar la problemática ambiental a través de sus diferentes escalas de análisis espacial y temporal. De esta manera es posible problematizar la realidad ambiental desde la perspectiva local considerando sus cambios dinámicos a través del tiempo (Gonzalez del Solar \& Marone 2001, Bermudez \& De Longhi 2008). Comunicar el conocimiento ecológico y que éste sea apropiado por la sociedad propicia que los miembros de la comunidad vean estimuladas sus perspectivas críticas y contribuyan con bases firmes a fortalecer su autonomía y la discusión ambiental (Gómez 1995), así como al desarrollo de los mecanismos para generar, responder y validar las diferentes preguntas en torno al tema (Mayer 1998, Farji-Brener 2007, González Espada 2007, Blumstein \& Saylan 2007).

En Sudamérica, y en el Uruguay en particular, se observa en los últimos años un incremento de bibliografía sobre EA y desarrollo sustentable (e.g. Achkar et al. 2004, Gudynas 2004, Achkar et al. 2005a, 2005b, 2006, 2007), proyectos que combinan desde distintos ámbitos la participación de las ONG's con la Universidad, así como la realización de encuentros y la conformación de diversas redes de trabajo en el área. Donde la EA es una herramienta clave para difundir las temáticas ambientales y promover la adopción de enfoques multidisciplinarios y participativos.

En este sentido la ecología puede permear a través de la EA y contribuir mejor en el manejo ambiental y la conservación (Bermudez \& De Longhi 2008). La efectividad de este proceso puede verse reflejada con la apertura de nuevos espacios en congresos de especialistas, los que en sus programas incorporan talleres especiales para comunicar diversas experiencias educativas. Además, algunas organizaciones combinan la investigación en conservación con EA y trabajan con las comunidades locales donde realizan sus investigaciones. De esta forma los ciudadanos que participan en las actividades de EA son informados e interactúan de diversas maneras involucrándose con las investigaciones y compartiendo los objetivos de conservación (Jacobson 2006).

Sin embargo existen dificultades a la hora de transferir el conocimiento científico a la sociedad en general y en particular a la comunidad educativa (Solarte 2006). Transformar el conocimiento científico en una versión comprensible que pueda ser enseñable, requiere de su extracción e inserción en un discurso didáctico, un proceso denominado transposición didáctica (Chevalard 1985). Una manera de acercar el conocimiento científico y que este pueda ser transformado y adaptado por el educador, que es quien maneja y utiliza los modelos pedagógicos, es a través de portales educativos interactivos en Internet.

En Uruguay se encuentra en funcionamiento el proyecto CEIBAL "Conectividad Educativa de Informática Básica para el Aprendizaje en Línea”. El objetivo es proporcionar a todos los alumnos y maestros de las escuelas públicas del país, el acceso a la tecnología a través de la entrega gratuita de un ordenador portátil que niños y maestros llevan a sus casas y con los que trabajan en la escuela (Vázquez 2009). De esta forma la comunidad, a través del portal educativo "Plan Ceibal" (www.ceibal. edu.uy), accede a diversidad de contenidos educativos donde trabajar diversas temáticas y producir a su vez ellos mismos contenidos con énfasis en la situación local. Este plan se presenta como una oportunidad para que los científicos experimenten nuevas formas de comunicar el conocimiento. Y la adaptación de los contenidos puede resultar del trabajo conjunto entre científicos, docentes y estudiantes. En este marco el educador es quien logra transformar el saber científico a saber enseñable a través de la transposición didáctica (Falcão \& Silva 2007), siendo esencial la labor conjunta del científico al proporcionar el marco teórico vinculado a la situación local, el educador que es quien adapta estos contenidos en función de las necesidades reales de su comunidad educativa, y el que aprende incorporando los contenidos a sus esquemas cognitivos. Este enfoque propone un cambio en el vínculo teórico-práctico, donde se acerquen las necesidades sociales al quehacer científico y la práctica educativa.

A partir de esta vinculación entre ciencia y comunidad, y la oportunidad de contar con un 
recurso como el "Plan Ceibal", este manuscrito describe un trabajo cuyo objetivo fue realizar un estudio ecológico local, y a partir del conocimiento generado, plantear y proponer propuestas de actividades educativas ambientales, las cuales pudieran ser transferidas a la comunidad a través del uso del portal educativo. Para esto se estudió un ecosistema modificado por las actividades humanas que poseía interesantes potenciales para ser utilizado en el desarrollo de actividades con la comunidad local. Estas actividades se basarían en la interpretación de los procesos dinámicos que se realizan

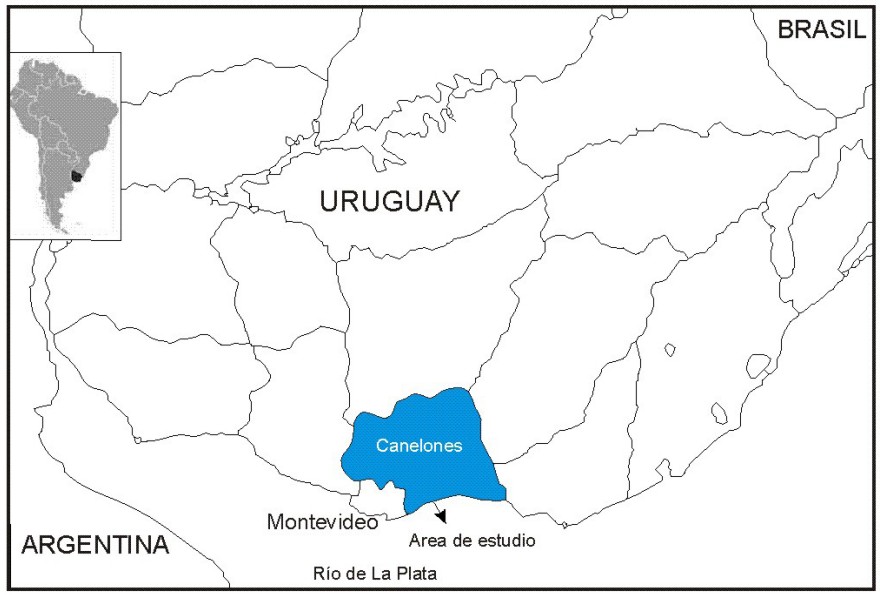

Figura 1 - Ubicación del área de estudio en el espacio geográfico en su conjunto, y cómo éstos se relacionan con las múltiples dimensiones del ambiente (Grizzle 1994, Artaraz 2002, Achkar et al. 2007). De esta forma el conocimiento de base que surgió a partir del estudio de ambientes o ecosistemas puede ser utilizado para la realización de actividades de EA que tiendan a mejorar la planificación ambiental y territorial así como la gestión de los recursos naturales de la zona con la participación de la comunidad (Meffe et al. 2006, Casado \& Alonso Mielgo 2007).

\section{Objetivo general}

A partir de datos científicos, obtenidos de una investigación ecológica en un área geográfica determinada, se proponen actividades de educación ambiental para la comunidad educativa local, especificando su implementación y socialización.

\section{Metodología}

El presente trabajo se desarrolló en la localidad turística y residente del balneario "El Pinar" ubicado en la zona costera del Río de la Plata en el departamento de Canelones-Uruguay (34 $48^{\circ} \mathrm{S}$, $\left.55^{\circ} 54^{\prime} \mathrm{W}\right)$ (Fig. 1). El balneario se integra a la "Ciudad de la Costa" que se extiende a lo largo de 17 kilómetros en el Departamento de Canelones. Posee un total de 17.221 habitantes según el último conteo de población del año 2004 (Intendencia Municipal de Canelones) y en las últimas tres décadas ha presentado un elevado crecimiento demográfico. Junto a este incremento ocurre un cambio en el uso del espacio geográfico, de ciudad balnearia hasta la década de 1970, para transformarse en ciudad dor- mitorio de Montevideo (Achkar et al. 2004). Esta situación de aumento en el proceso de urbanización y cambios estructurales en el uso del espacio trae como consecuencia importantes problemas ambientales, los cuales deberán ser enfrentados por la comunidad local (Beaujeu-Garnier \& Chabot 1970, Cunill Grau 1995, Morello et al. 1998, Brailovsky \& Foguelman 2004).

Se realizó un estudio ecológico en un área ubicada en el sistema de dunas costeras utilizando fotografías aéreas obtenidas del Servicio de Sensores Remotos Aeroespaciales de la Fuerza Aérea Uruguaya y del Servicio Geográfico Militar en una serie temporal de imágenes desde 1966 a 2001. A través de este estudio se reportó y cuantificó un aumento de la cobertura vegetal sobre el sistema de dunas costeras a lo largo del tiempo (originalmente poseía escasa vegetación), con una direccionalidad de avance hacia la línea de costa. Se analizó la importancia de los cambios en la estructura del paisaje como consecuencia de las modificaciones antrópicas, principalmente la forestación con especies exóticas y el desarrollo de la urbanización. Finalmente se realizó un muestreo de vegetación y se determinó la ubicación de diferentes formaciones vegetales.

A partir de la delimitación de esta área de estudio se consideró el área de influencia para desarrollar las actividades de EA. Se incluyeron centros educativos de educación primaria y secundaria, pública y privada. En algunos de estos centros educativos se realizaron entrevistas a maestros y docentes para conocer el tipo de actividades relacionadas al ambiente que llevaban a cabo con sus estudiantes. A pesar de la proximidad de los centros educativos al área costera, los maestros y docentes señalaron que la ausencia de espacios al aire libre resultaba ser una 
limitante para realizar tales actividades. Se identificaron y geo-referenciaron, a través del uso de un dispositivo de geo-posicionamiento satelital (GPS), los principales centros educativos de la "Ciudad de la Costa” (escuelas y liceos), los puntos de encuentros de la comunidad local y las principales rutas de acceso al área de estudio (Fig. 2).

Los resultados obtenidos en el estudio ecológico proporcionaron el marco teórico-práctico de conocimiento sobre el área de estudio, en base al cual se plantea una propuesta de desarrollo de actividades de EA siguiendo pautas establecidas para trabajos del área educativa (Vayer et al. 1993, Arango et al. 2002, Barderi et al. 2004, Biendell et al. 2006, de León \& Gasdía 2008, de Moura Carvalho 2008, Ressia 2008).

\section{Resultados}

El área de influencia contiene a los balnearios "Parque Carrasco", "Shangrilá”, "San José de Carrasco", "Lagomar", "Solymar", "Parque de Solymar", "Lomas de Solymar", "Medanos de Solymar" y "El Pinar". Incluye 25 centros educativos repartidos en un total de 20 escuelas y 5 liceos, una comisión de fomento y una ONG conservacionista (Fig. 2). A partir de los resultados obtenidos del estudio ecológico se asoció la información cuantitativa con las actividades de propuestas educativas.

\section{Actividad 1: Dinámica del espacio geográfico y noción de escala temporal}

\section{Objetivo educativo}

Comprender el uso y la organización del espacio que realiza la comunidad en la complejidad de sus actos sociales a lo largo de un proceso histórico.

\section{Objetivo práctico}

Interpretar los procesos dinámicos que se producen en el espacio geográfico a través de fotografías aéreas históricas.

A partir de las fotos aéreas de los años 1966, 1976, 1982, 1987, 1994 y 2001, obtenidas, se propone generar cartografía que contenga las características urbanas (e.g. rutas principales, calles, caminos vecinales, casas e infraestructuras), de usos de suelo (e.g. agrícola, ganadero, forestal) y del ecosistema (e.g. suelo, vegetación) (Barderi et al. 2004). En base a la superposición de la cartografía se sugiere realizar un análisis espacial secuenciado con el fin de identificar y comprender la evolución temporal del espacio geográfico, identificando y localizando los bienes ambientales (Morello et al. 1998). El resultado del análisis de la serie temporal de la cartografía reportará la evolución temporal del paisaje, el cambio de la estructura del mismo y el aumento de las modificaciones antrópicas (Biendell et al. 2006).

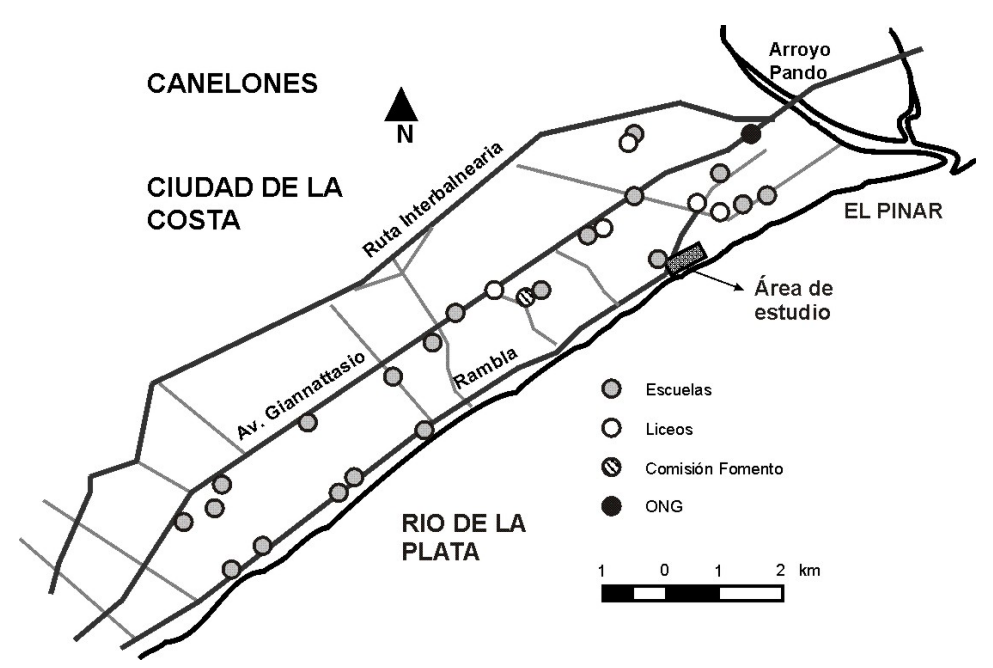

Figura 2 - Mapa del área de influencia para el área de estudio donde se ubican los principales centros educativos (Escuelas y Liceos)

\section{Actividad 2: Interpretación} ecosistémica

\section{Objetivo educativo}

Planificación de una tarea práctica de campo que incluya la sistematización de datos y la visualización de la heterogeneidad ambiental in situ.

\section{Objetivo práctico}

Lectura de un paisaje y descripción de los componentes físicobiológicos que lo componen.

Se propone comenzar con la 
observación de la topografía lo que implica determinar las zonas de dunas y las zonas inter-dunares. A partir de esta delimitación se propone tomar muestras para evaluar el tipo de suelo, contenido de materia orgánica y humedad, y reportar qué proporción del área de estudio se encuentra cubierta por arena y cuál por vegetación. Es posible correlacionar la localización de los diferentes tipos de suelo, y el contenido de humedad, con la topografía y la presencia-ausencia de vegetación. La descripción de la vegetación a través del reporte de árboles, arbustos y herbáceas, asî como la identificación y observación del tipo de hojas, raíces y rizomas, puede ser utilizada para relacionar adaptaciones vegetales y características del sitio. La abundancia y riqueza de especies para cada ambiente puede ser registrada utilizando el método de parcelas, la lista de especies para el área de estudio, y bibliografía complementaria (Lombardo 1982, 1983, 1984, Alonso Paz \& Bassagoda 1999, Arango et al. 2002, Fagúndez \& Lezama 2005, Alonso Paz 2006).

\section{Actividad 3: Ambiente y participación}

\section{Objetivo educativo}

Promover la participación en la identificación, discusión y diagnóstico de problemas y conflictos ambientales locales así como sus posibles soluciones.

\section{Objetivo práctico}

Realizar una identificación y un diagnostico de los problemas y conflictos ambientales.

Se propone utilizar la información geográfica representada en los mapas para identificar, discutir y diagnosticar los problemas y posibles conflictos locales. Mediante una reconstrucción histórica del proceso de urbanización y la realización de entrevistas y encuestas a vecinos y técnicos, es posible comprender parte de la configuración del espacio social y como éste interactúa con la naturaleza. Un ejemplo es establecer las formas de apropiación/ valoración social de los elementos de la naturaleza. Cuando se involucran grupos sociales con intereses y visiones contrapuestos, pueden existir conflictos relacionados a su gestión y apropiación. Se propone la identificación de grupos que luchan por un uso y acceso legítimo de los bienes naturales, la identificación de formas diferentes de interacción con la naturaleza y del conocimiento local (e.g. pescadores artesanales, mujeres que rescatan conocimientos populares sobre las plantas medicinales, etc.) y otros grupos con intereses que pueden comprometer la calidad de tales bienes o su desaparición (de Moura Carvalho 2008).

\section{Actividad 4: Redes de conocimiento}

\section{Objetivo educativo}

Comprender las distintas etapas y procesos ligados a la producción de conocimiento y profundizar en los aspectos relacionados a su democratización y a cómo el conocimiento puede ser utilizado/apropiado en el seno de una red social que promueva acciones concretas referidas a lo socio-ambiental.

\section{Objetivo práctico}

Realizar una síntesis de las actividades y resultados para ser compartidos online con la comunidad educativa.

El medio online de aprendizaje, gracias a las potencialidades interactivas de los ordenadores e Internet, permite a los discentes la comunicación personalizada y colaborativa en red (Santos \& Silva 2009). De esta manera se consigue compartir la información, experiencias, resultados y opiniones de manera atractiva a través de la digitalización de contenidos en diversos formatos, como textos, fotografías, videos y sonidos. Las experiencias recogidas en un centro educativo en particular pueden vincularse a otras experiencias, en centros geográficamente distantes, y elaborar colectivamente un nuevo conocimiento no acabado, que fortalezca a las redes sociales involucradas con la problemática ambiental y sus soluciones.

\section{Discusión de los Resultados}

El presente trabajo se sustenta en la vinculación de los resultados obtenidos a través de una investigación ecológica, con propuestas educativas que se ofrecerán a la comunidad a través del portal educativo del "Plan Ceibal" generando una nueva forma de presentary difundir el conocimiento científico; conocimiento que pretende contribuir a la enseñanza ambiental. Es una propuesta que busca relacionar la teoría y práctica ecológica y las actividades de EA para contribuir a una concepción 
amplia del ambiente, donde se considere el funcionamiento e interrelaciones de los componentes bióticos y abióticos y al ser humano como parte de las comunidades bióticas (Grizzle 1994, McComas 2002, Bermudez \& De Longhi 2008).

Es por esto que un ecosistema costero como el observado en "El Pinar" (Canelones), que ha sufrido intensas modificaciones, es un buen ejemplo para vincular y estudiar el efecto de las actividades antrópicas en el ambiente y la respuesta de las comunidades biológicas. En el estudio ecológico se evidenció la transformación de un paisaje con predominio de dunas y escasa vegetación, a un ambiente modificado donde se generan condiciones para un mayor establecimiento de comunidades vegetales. Este punto puede resultar útil para discutir la importancia del efecto antrópico en la construcción de neo-ecosistemas (Jordan III et al. 2003, Meffe et al. 2006). De esta forma se puede contribuir a disminuir la percepción de que el ser humano no es integrante natural de los ecosistemas, y la idea de que su interacción sólo causa impactos negativos. La consideración a priori de que un aumento de la vegetación puede considerarse como un impacto positivo es el punto de partida para estas consideraciones (Grizzle 1994). Ahora bien, el aumento de la vegetación en un ecosistema costero no se traduce necesariamente en que la modificación sea positiva, ya que ésta puede afectar la dinámica de la arena y contribuir al retroceso de la línea de costa (Hesp 2002). Por lo que este caso es un buen ejemplo de la complejidad que presentan los sistemas ambientales, y a través del conocimiento de base generado se puede realizar una lectura crítica, esencial para contribuir a la discusión ambiental. Mediante este abordaje de construcción crítica, y al integrar al ser humano como parte del sistema ambiental, se busca un resultado a largo plazo que contribuya a los esfuerzos de conservación y sustentabilidad ambiental (Grizzle 1994, de Souza Pinto Cribb 2008).

Las actividades propuestas enfatizan en un principio en cómo las comunidades humanas utilizan, organizan, y construyen el espacio de forma dinámica a lo largo de un proceso histórico, incorporando como ejes temáticos la temporalidad y la espacialidad, de particular importancia en los estudios ecológicos (Actividad 1) (Levin 1992). Posteriormente se plantean actividades para generar un conocimiento sistematizado y manejable a través de mapas, con los cuales analizar la heterogeneidad ambiental (Turner et al. 2001). Las tareas prácticas de campo permiten contrastar hipótesis y desarrollar los sentidos exploratorios que estimulan un mejor relacionamiento con el ambiente (Actividad 2) (Arango et al. 2002). Y en la misma esfera la realización de entrevistas y encuestas lleva a la participación de toda la comunidad en la identificación, discusión y diagnóstico de problemas y conflictos ambientales locales así como sus posibles soluciones (Actividad 3). La utilización de las tecnologías de información y comunicación, como en este caso las computadoras del "Plan Ceibal", pueden potenciar la apropiación de la tecnología como herramienta para fortalecer los vínculos en red de estas comunidades escolares "generadoras de conocimiento" (Actividad 4). Su utilización para trabajar y difundir las realidades y problemáticas locales es también un elemento importante de la EA. Se trata de un énfasis en la forma del aprendizaje y no sólo en los contenidos. El aprendizaje mejora si responde a las necesidades y situación del que aprende y si se estimula un aprendizaje significativo, experimental y participativo (Orr 1992).

\section{Agradecimientos}

Este trabajo es dedicado y en memoria de Elena Latorre.

\section{Referencias bibliográficas}

Achkar M., Domínguez A., Pesce F. 2004. Diagnóstico socioambiental participativo en Uruguay. Montevideo: Tomate Verde. 157p.

Achkar M., Domínguez A., Pesce F. 2005a. Educación para la sustentabilidad. Quehacer educativo. 37-39.

Achkar M., Domínguez A., Pesce F. 2005b. Educación, ambiente y desarrollo sustentable: una tríada forjada a lo largo de tres décadas. Quehacer educativo. 34-42.

Achkar M., Domínguez A., Pesce F. 2006. La temática ambiental en el aula: contenidos a abordar. Quehacer educativo. 44-48.

Achkar M., Domínguez A., Pesce F. 2007. Educación Ambiental. Una demanda del mundo de hoy Montevideo: Tomate Verde. 141p.

Alonso Paz E. 2006. Flora y vegetación de la costa platense y atlántica uruguaya. In: Menafra R. RodríguezGallego L. Scarabino F. \& Conde D. eds. 2006. Bases para la conservación yel manejo de la costa uruguaya. Montevideo: Vida Silvestre Uruguay. p. 71-88.

Alonso Paz E., Bassagoda M.J. 1999. Los bosques y los matorrales psamófilos en el litoral platense y atlán- 
tico del Uruguay. Comunicaciones botánicas del Museo de Historia Natural de Montevideo, VI(113):8.

Arango N., Cháves M.E., Feinsinger P. 2002. Enseñanza de ecología en el patio de la escuela: guía metodológica para la enseñanza de ecología en el patio de la escuela. New York: National Audubon Society. $92 \mathrm{p}$.

Arocena R., Bortagaray I., Sutz J. 2008. Reforma universitaria y desarrollo. Montevideo: Tradinco. 256p.

Artaraz M. 2002. Teoría de las tres dimensiones de desarrollo sostenible. Ecosistemas. (3):1-7.

Barderi M.B., Carminati A., Fernández Balboa C.G., Franco R., Wall L.G. 2004. Biología: las relaciones de los seres vivos entre sí y con su ambiente, 1a edición. Buenos Aires, Argentina: Ed. Santillana S.A. 207p.

Barreiro J., Gatto H., Gudynas E., Honty G., Leff E., Mora E., Santandreu A. 1996. Democracia y ecología. La política de la gestión ambiental. Montevideo. $144 \mathrm{p}$.

Beaujeu-Garnier J., Chabot G. 1970. Tratado de geografía urbana. Editorial Vicens-Vives. 587p.

Bermudez G., De Longhi A.L. 2008. La educación ambiental y la ecología como ciencia. Una discusión necesaria para la enseñanza. Revista electrónica de enseñanza de las ciencias, 7(2):275-297.

Biendell A.A., Casalis B., Corazza J.R., Gamarra M.B., Milani M.T., Orellano H.A., Tórtolo V.M. 2006. Nuestra comunidad a través de los testimónios: desde el pasado remoto hasta nuestros días. Córdoba, Argentina: Fundación AGM. 104p.

Blumstein D.T., Saylan C. 2007. The failure of environmental education (and how we can fix it). Plos Biology. 5(5):0001-0005.

Bondolfi A. 2001. Ética del ambiente natural, derecho y políticas ambientales: tentativa de un balance y de perspectivas para el futuro. Acta Bioethica, 2:293-315.

Brailovsky A.E., Foguelman D. 2004. Memoria verde: historia ecológica de la Argentina, 24a edición. Buenos Aires, Argentina: Editorial Sudamericana S.A. 351p.

Bustillo L., Martínez J.P. 2008. Los enfoques del desarrollo sustentable. Interciencia, 33(5):389-395.

Casado G.I.G., Alonso Mielgo A.M. 2007. La investigación participativa en agroecología: una herramienta para el desarrollo sustentable. Ecosistemas, 16(1):24-36.

Chevalard Y. 1985. La transposition didactique: du savoir savant au savoir enseigné. Paris: La Penseé sauvage. $196 \mathrm{p}$.

Costanza R. 2000. Visions of alternative (unpredictable) futures and their use in policy analysis. In: Conservation ecology, URL: http://www.consecol.org/vol4/ iss1/art5/. Acesso: 11.12.2008.

Cunill Grau P. 1995. Las transformaciones del espacio geohistórico latinoamericano 1930-1990, 1a edición. DF, Mexico: Fideicomiso Historia de las Amé- ricas. $198 \mathrm{p}$.

de León M.J., Gasdía V. 2008. Biodiversidad del Uruguay. Montevideo, Uruguay: Editorial Fin de Siglo. 270p.

de Moura Carvalho I.C. 2008. Educaçao ambiental: a formaçao do sujeito ecológico. 3a edición. São Paulo: Cortez Editora. 256p.

de Souza Pinto Cribb S.L. 2008. Gestão das áreas de proteção ambiental e comunidades locais: uma parceria necessária. Rev. Eletr. Mestr. Educ. Ambient., 20:350-371.

Fagúndez C., Lezama F. 2005. Distribución espacial de la vegetación costera del litoral platense y atlántico uruguayo. Uruguay: Informe Freplata. 36p.

Falcão E.B.M., Silva F.F. 2007. Os sentidos de natureza na formação e na prática científica. Educação e Pesquisa, 33, 369-385.

Farji-Brener A. 2007. Una forma alternativa para la enseñanza del método hipotético-deductivo. Interciencia, 32(10):716-720.

Feldmann F.J., Biderman Furriela R. 2001. Los cambios climáticos globales y el desafío de la ciudadanía planetaria. Acta Bioethica, 2:287-292.

Ferreira L., Pereira da Silva M., Kelton J., Vasconcelos C. 2007. Educacao ambiental em comunidades rurais de Juazeirinho-PB: Estratégias e desafios. Rev. Eletrônica Mestr. Educ. Ambient., 19:110-121.

Gómez M. 1995. Investigación educativa y polémica en America Latina. In: Puiggrós A. \& Gómez M. eds. 1995. Alternativas pedagógicas, sujetos y prospectiva de la educación latinoamericana. Instituto de Investigaciones en Ciencias de la Educación. Facultad de Filosofía y Letras. Universidad de Buenos Aires, Buenos Aires. p. 17-35.

Gonzalez del Solar R., Marone L. 2001. The "freezing" of science: Consequences of the dogmatic teaching of ecology. BioScience, 8(51):683-686.

González Espada W.J. 2007. The role of the scientific community in school science education. Interciencia, 32(8):510-515.

González García M.I., López Cerezo J.A., Luján López J.L. 1996. Ciencia, tecnología y sociedad: una introducción al estudio social de la ciencia y la tecnología. Madrid: Tecnos. 120p.

González Gaudiano E. 2001. Otra lectura a la historia de la educación ambiental en América Latina y el Caribe. Desenvolvimento e Meio Ambiente, (3):141-158.

Gough N. 2002. Thinking/acting locally/globally: Western science and environmental education in a global knowledge economy. International Journal of Science Education, 24(11):1217-1237.

Grizzle R.E. 1994. Environmentalism should include human ecological needs. BioScience, 44(4):263-268.

Gudynas E. 2004. Ecología, economía y ética del desarrollo sostenible. Montevideo: Centro Latinoamericano de Ecología Social. 260p.

Gutiérrez J., Pozo T. 2005. Stultifera Navis: institu- 
tional tensions, conceptual chaos, and professional uncertainty at the beginning of the Decade of Education for Sustainable Development. Policy Futures in Education, 3:296-308.

Gutiérrez J., Pozo T. 2006. Modelos teóricos contemporáneos marcos de fundamentación de la educación ambiental para el desarrollo sostenible. Revista Iberoamericana de Educación, 41:21-68.

Hesp P. 2002. Foredunes and blowouts: initiation, geomorphology and dynamics. Geomorphology, 48:245-268.

Jacobson S.K. 2006. The Importance of Public Education for Biological Conservation. In: Groom M.J. Meffe G.K. \& Carroll C.R. eds. 2006. Principles of Conservation Biology. Massachusetts, U.S.A.: Sinauer Associates, Inc. p. 681-683.

Jordan III W.R., Gilpin M.E., Aber J.D. 2003. Restoration ecology: A synthetic approach to ecological research. Cambridge. Cambridge Univ. Press. 342p.

Kates R.W., Parris T.M. 2003. Long-term trends and a sustainability transition. Proceedings of the National Academy of Sciences, 100(14):8062-8067.

Levin S. 1992. The problem of pattern and scale in ecology. Ecology, 73:1943-1967.

Lombardo A. 1982. Flora Montevidensis. Montevideo: Intendencia Municipal de Montevideo. Servicio de Publicaciones y Prensa. 316p.

Lombardo A. 1983. Flora Montevidensis. Gamopetalas. Montevideo: Intendencia Municipal de Montevideo. Servicio de Publicaciones y Prensa. 347p.

Lombardo A. 1984. Flora Montevidensis. Monocotiledoneas. Montevideo: Intendencia Municipal de Montevideo. Servicio de Publicaciones y Prensa. 465p.

Martínez Alier J. 1995. De la economía ecológica al ecologismo popular. Ecoteca. Nordan-Comunidad. Icaria. 286p.

Mayer M. 1998. Educación ambiental: de la acción a la investigación. Enseñanza de las Ciencias, 16(2): 217-231.

McComas W.F. 2002. The ideal environmental science curriculum: I. history, rationales, misconce. The American Biology Teacher, 9(64):665-672.

Meffe G.K., Groom M.J., Carroll C.R. 2006. Ecosystem approaches to conservation. Responses to a complex world. In: Meffe G.K. Groom M.J. \& Carroll C.R. eds. 2006. Principles of conservation biology. Massachusetts: Sinauer Associates, Inc. p. 468-507.

Morais E., Silva F. 2007. Os sentidos de natureza na formação e na prática científica. Educação e Pesquisa, 33(2):369-385.
Morello J., Matteucci S.D., Buzai G.D., Baxendale C., Rodríguez A. 1998. Aplicación de la tecnología SIG para el análisis del soporte biofísico en áreas metropolitanas como herramienta de planificación. El caso de Buenos Aires. In: Matteucci S.D. \& Buzai G.D. eds. 1998. Sistemas ambientales complejos: herramientas de análisis espacial. Buenos Aires: Editorial Universitaria de Buenos Aires. p. 409-423.

Orr D.W. 1992. Ecological literacy: education and the transition to a postmodern World. Albany: S.U.N.Y. Press.

Porlán R. 1999. Hacia un modelo de enseñanzaaprendizaje de las ciencias por investigación. In: Kaufman M. \& Fumagalli L. eds. 1999. Enseñar ciencias naturales. Reflexiones y propuestas didácticas. México: Paidós Educador. p. 23-64.

Ressia G.A. 2008. 100 ideas para la práctica de la educación ambiental: recursos para el aula, 1a edición. Buenos Aires, Argentina: Editorial Troquel S.A. $125 \mathrm{p}$.

Santos E., Silva M. 2009. O desenho didático interativo na educação online. Revista Iberoamericana de Educación, (49):267-287.

Sauvé L. 1999. La educación ambiental entre la modernidad y la posmodernidad: en busca de un marco de referencia educativo integrador. Tópicos en Educación Ambiental, 2(1):7-25.

Solarte M.C. 2006. Los conceptos científicos presentados en los textos escolares: son consecuencia de la transposición didáctica. Revista Electrónica de la Red de Investigación Educativa, 1(4):1-12.

Turner M.G., Gardner R.H., O'Neill R.V. 2001. Landscape ecology in theory and practice, pattern and process. USA: Springer. 401p.

UN. 1987. Our Common Future. In Report of the World Commission on Environment and Development. URL: http:/www.un-documents.net/ wced-ocf.htm. Acesso: 22.6.2009

UNESCO-PNUMA. 1975. Seminario Internacional de Educación Ambiental. In: Programa Internacional de Educación Ambiental. URL: http://unesdoc. unesco.org/images/0002/000276/027608sb.pdf. Acesso: 22.6.2009

UNESCO. 1977. Tbilisi Declaration. URL : http:// www.gdrc.org/vem/ee/tbilisi.html. Acesso: 23.6.2009

Vayer P., Duval A., Roncin C. 1993. na ecología de la escuela: la dinámica de las estructuras materiales. Barcelona: Ediciones Paidos. 205p.

Vázquez T. 2009. Digital Democracy. In: Americas Quarterly. URL: http://www.americasquarterly. org/node/370. Acesso: 4.3.2009 